\title{
A Large Infantile Capillary Hemangioma of the Eyelid with 5 Years Follow-up
}

Mohammad Idris1, Mahfooz Hussain1, Junaid Sethi1, Zubairullah Khan² and Muhammad Zubair Umer³

\begin{abstract}
A five-day baby girl presented with a large mass involving the right upper and lower eyelid obstructing the visual axis completely. She was treated with intralesional triamcinolone acetonide injection under general anesthesia but no change in size of the tumor was observed. Oral beta blocker, propranolol, $1 \mathrm{mg} / \mathrm{kg}$ body weight, was given at three months. After two months, no significant change in tumor size was seen. The tumor was managed by surgical excision. There was no effect of intralesional triamcinolone acetonide and oral beta blockers. Even with surgical excision, there was recurrence of tumor.
\end{abstract}

Key Words: Periocular capillary hemangioma, Beta blocker, Steroids.

\section{INTRODUCTION}

Capillary hemangioma of the orbit and periorbital region is very common tumor of childhood. Different treatment modalities aiming at early removal of the tumor are available including intralesional steroids, beta blockers and surgery. ${ }^{1}$ It usually appears as small isolated lesion. Observation is the rule initially, as most of the time spontaneous involution is seen and no sequalae are seen in majority of the cases. ${ }^{2}$

In rare cases, they appear as large masses without spontaneous resolution. Such rare cases are responsible for stimulus-deprivation amblyopia. We report a case of large infantile periocular capillary hemangioma of upper eyelid in which neither steroids nor beta blockers were effective.

\section{CASE REPORT}

A five-day baby girl presented with a large mass involving the right upper and lower eyelids obstructing the visual axis completely. It was present since birth, which increased in size thereafter. On examination, it was a well-demarcated, fleshy dark red mass measuring $2 \times 2 \times$ $0.8 \mathrm{~cm}$ in size. Entire upper eyelid was covered and the overlying skin had reddish discoloration (Figure 1). It was soft, non-pulsatile and non-reducible, which blanched on pressure. On palpation, it was very fragile.

I Department of Ophthalmology, Lady Reading Hospital/MTI, Peshawar, Pakistan

2 Department of Ophthalmology, Frontier Medical College, Abbottabad, Pakistan

3 Department of Pathology, Khyber Medical College, Peshawar, Pakistan

Correspondence: Dr. Mohammad Idris, Eye Unit, Lady Reading

Hospital/MTI, Peshawar, Pakistan

E-mail: idrisdaud80@gmail.com

Received: January 01, 2018; Accepted: July 30, 2018
Its size increased on crying and there was no bruit noted.

There were no significant problems in the baby besides the periorbital tumor on examination. The other eye was completely normal. The blood profile was normal. Treatment was started with a single intralesional injection of triamcinolone acetonide, $0.4 \mathrm{mg}$ in $0.1 \mathrm{ml}$, followed a month later by oral beta blocker, propranolol, $1 \mathrm{mg} / \mathrm{kg}$ body weight, after consultation with a pediatrician. The baby's response to treatment was noted at 4 weeks, and there was no reduction in the size of the lesion. There was history of bleeding as noted by the parents; and they insisted for surgical removal. Surgery was undertaken at 18 months of age. The lesion was excised under general anesthesia. Fortunately, there was enough skin available for reapproximation of the defect, so 6/0 vicryl, suture was used after properly securing bleeding with cautery. It was surprisingly noted that bleeding was not very significant. The baby was followed at one month (Figure 2) and the biopsy report revealed capillory hemangioma.

At one year of follow-up, slight swelling of the right upper eye was noted, representing a recurrence, so it was excised again; but there was significant bleeding on the table. At 5 years follow-up, no recurrence was noted (Figure 3).

\section{DISCUSSION}

We have an infant of 5 days with large right eyelid tumor. Its size was more than $2 \mathrm{~cm}$. It was red, strawberry shaped, typical of capillary hemangioma. ${ }^{3}$ Capillary hemangioma usually has an initial "proliferative" phase in which rapid growth occurs within 3-6 months. In involution phase, the tumor regresses. ${ }^{4}$ However, in this case, according to parents of the baby, within few days of birth, it underwent very rapid growth and developed into a large mass covering the visual axis completely. 


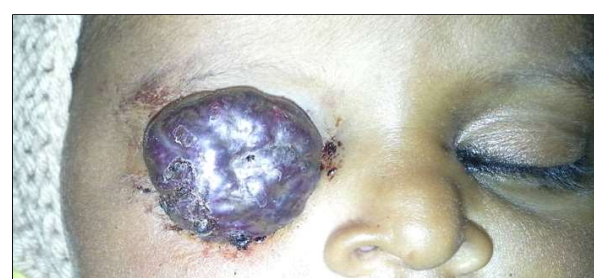

Figure 1: Tumor covering both the eyelids of the right eye.

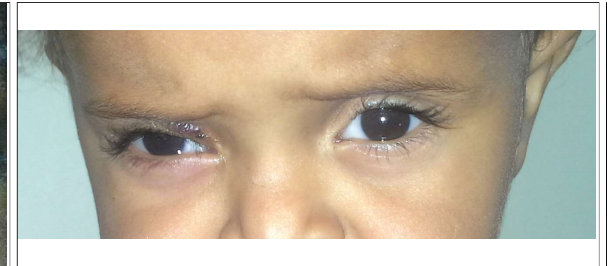

Figure 2: Photograph of child (18 months) after one month surgery.
Infantile hemangiomas are a common vascular tumor of infancy with heterogeneous presentations and unique growth features. If the size is large enough to occlude the visual axis, it can lead to amblyopia. A tumor size of more than $1 \mathrm{~cm}$ is an important cause of amblyopia with $50 \%$ of such patients developing amblyopia. ${ }^{5}$ The tumor size in this case was $2 \mathrm{~cm}$. Intralesional steroid treatment was tried. A study reported the use of intralesional steroid as the most common initial therapy without serious complications. ${ }^{6}$ This therapy was tried initially followed by beta blockers which did not work in reducing the size of the lesion and clearing of the visual axis. Then a surgical excision was planned of the whole tumor with full consent of parents for risk of bleeding but unexpectedly, there was little bleeding on the table. Tumor was removed and lid skin edges were approximated with $6 / 0$ vicryl suture. At one year, she presented with small tumor regrowth. It was again excised but this time, there was copious bleed on the table which required extensive resuturing. This was a challenging case of capillary hemangioma. At 5-year follow-up, no recurrence was noted.
Intralesional injection of steroids as well as use of systemic beta blockers are ineffective in larger lesions of infantile periocular capillary hemangiomas. There are chances of recurrence even after surgery in rare and challenging cases.

\section{REFERENCES}

1. Cheng JF, Gole GA, Sullivan TJ. Propranolol in the management of periorbital infantile haemangioma. Clin Exp Ophthalmol 2010; 38:547-53.

2. Finn MC, Glowacki J, Mulliken JB. Congenital vascular lesions: clinical application of a new classification. J Pediatr Surg 1983; 18:894-900.

3. Hernandez JA,Chia A, Quah BL, Seah LL. Periocular capillary hemangioma: management practices in recent years. Clin Ophthalmol 2013; 7:1227-32.

4. Spence-Shishido AA, Good WV, Baselga E, Frieden IJ. Hemangiomas and the eye. Clin Dermatol 2015; 33:170-82

5. Schwartz SR, Blei F, Ceisler E, Steele M, Furlan L, Kodsi S. Risk factors for amblyopia in children with capillary hemangiomas of the eyelids and orbit. J AAPOS 2006; 10:262-8

6. Orawiec B, Stefanczyk L, Czajkowski J, Bogorodzki B, Gralek M. Periorbital capillary hemangiomas in children--treatment and monitoring of treatment. Klin Oczna 1996; 98:217-20. 